\title{
Fetal descending aortic flow and heart rate monitoring with transesophageal echocardiography during maternal cardiac surgery
}

\author{
Xiaodong Ye, MB $\cdot$ Yi He, MD $\cdot$ Zhengyuan Xia, MD, PhD $\cdot$ Sheng Wang, PhD
}

Received: 18 August 2015/Revised: 13 October 2015/Accepted: 22 October 2015/Published online: 30 October 2015

(C) The Author(s) 2015. This article is published with open access at Springerlink.com

\section{To the Editor,}

Herein, we report our experience with the use of transesophageal echocardiography (TEE) to monitor fetal heart rate in an advanced term pregnant patient undergoing cardiac surgery.

Cardiac diseases occur in approximately $2-4 \%$ of pregnancies. ${ }^{1}$ While maternal mortality rates have improved to levels similar to those in their non-pregnant counterparts, fetal mortality rates remain high when pregnant women undergo cardiac surgery utilizing cardiopulmonary bypass (CPB). ${ }^{2}$ Factors leading to high fetal mortality rates are variable, but the presence of fetal hypoxia, which is often reflected as sudden changes in intraoperative fetal heart rate (FHR), could be a major risk factor. Therefore, precise and timely monitoring of FHR is critical to optimizing fetal safety during maternal cardiac surgery.

We present a novel case where fetal descending aortic flow was monitored and converted to FHR by using TEE in a 26-yr-old female $(62 \mathrm{~kg})$ at 27 weeks of pregnancy undergoing double-valve replacement surgery. This patient had presented with swelling of her lower extremities and shortness of breath that progressed over a two-week period

X. Ye, MB · Y. He, MD · S. Wang, PhD ( $ه)$

Guangdong Cardiovascular Institute \& Guangdong General

Hospital, Guangdong Academy of Medical Sciences,

Guangzhou, Guangdong Province, China

e-mail: shengwang_gz@163.com

\section{Ye, MB}

Shantou University Medical College, Shantou, Guangdong Province, China

Z. Xia, MD, PhD

Department of Anesthesiology, The University of Hong Kong, Hong Kong, P.R China and was subsequently diagnosed with severe mitral stenosis, severe tricuspid regurgitation, severe pulmonary hypertension, and congestive heart failure. As the patient did not want an early termination of pregnancy, she was scheduled for mitral and tricuspid valve replacement with intraoperative FHR monitoring by TEE.

After anesthesia induction, a TEE probe was inserted and positioned at the deep gastric level. The TEE probe was then rotated until the fetal vertebral column was identified. With an upward $60-130^{\circ}$ rotation of the omniplane probe, signals from the fetal descending aortic flow were detected using pulsed-wave Doppler at a frequency of $4.4 \mathrm{MHz}$ and a depth of $14 \mathrm{~cm}$ (Figure, upper panel). The flow velocity was recorded and converted to FHR using the following formula:

The FHR $=60 \mathrm{sec} /$ the time interval between two adjacent peaks on the fetal descending aortic flow pulsedwave Doppler signal (sec).

The FHR was found to be $125-140$ beats $\cdot \mathrm{min}^{-1}$ with a regular rhythm before CPB (Figure, lower panel).

Before the initiation of CPB, 15,600 units of heparin were administered to achieve an activated clotting time $>$ $480 \mathrm{sec}$. Systemic normothermic CPB was established, and rectal temperature was maintained at $35.1-36.4^{\circ} \mathrm{C}$. On the initiation of $\mathrm{CPB}$, the FHR by TEE suddenly decreased to 80-100 beats $\mathrm{min}^{-1}$. Eight minutes after starting CPB, the FHR reached its lowest level of 77 beats $\cdot \mathrm{min}^{-1}$. By increasing the CPB pump flow to $>5.0 \mathrm{~L} \cdot \mathrm{min}^{-1}$, the FHR increased within minutes to $120-150$ beats $\min ^{-1}$. The operation continued without incident, and bioprosthetic valves were subsequently implanted in the mitral and tricuspid positions. The patient was then transferred to the cardiac intensive care unit where she stayed for three days with intermittent FHR monitoring using a transabdominal ultrasound monitor. Fetal heart rate was sustained at 

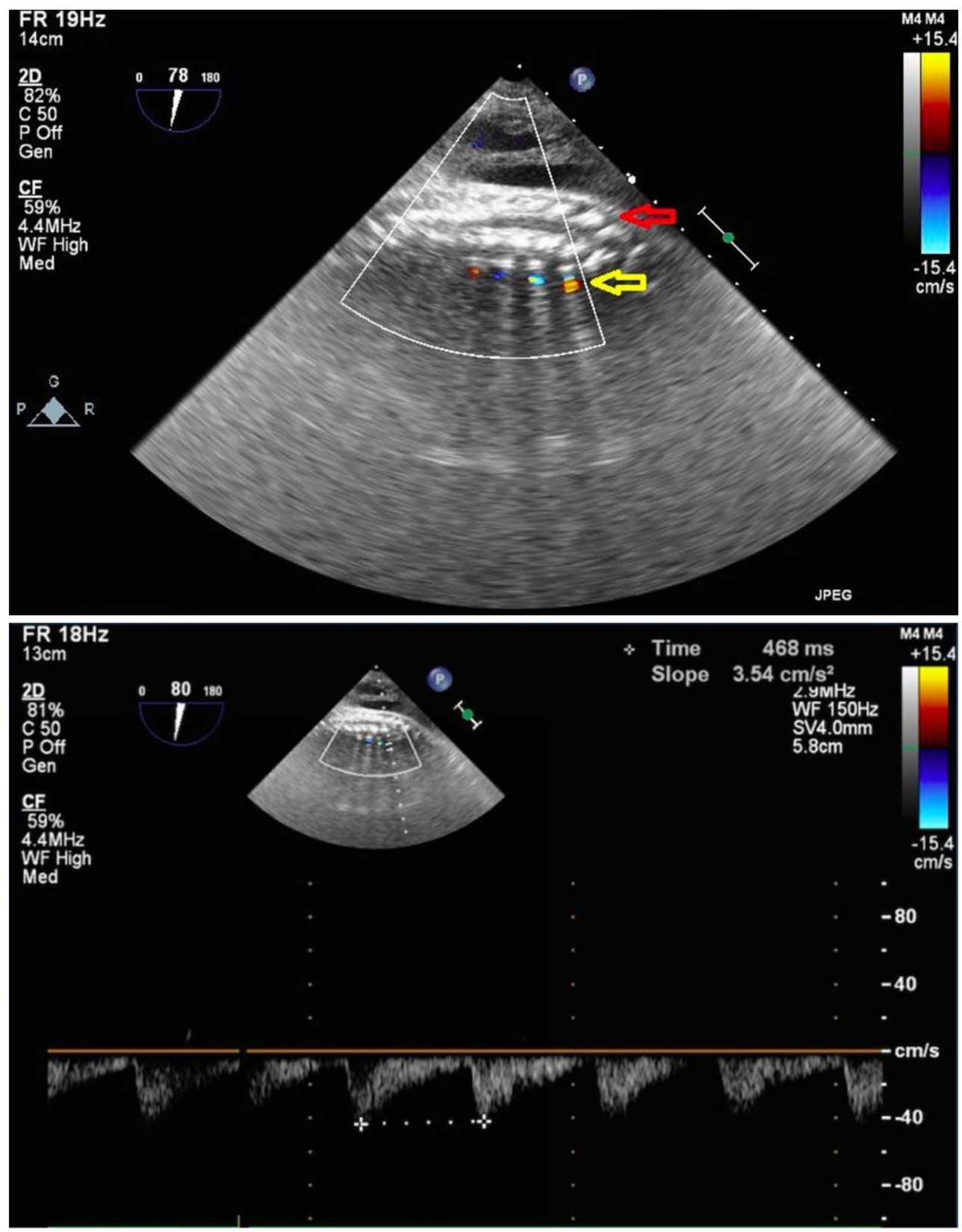

Figure A maternal transesophageal echocardiographic image of the fetal vertebral column of a 26-week fetus in a pregnant patient undergoing cardiac surgery. The red arrow shows the fetal vertebral column, and the yellow arrow shows the pulsed-wave Doppler signal

120-130 beats $\cdot \min ^{-1}$. She was subsequently transferred to the general ward where she remained for another ten days with intermittent FHR monitoring as deemed necessary. She was then discharged in good general condition and had a subsequent uneventful term delivery.

Fetal heart rate monitoring has been the most widely applied modality to evaluate the risk of fetal hypoxia. There have been previous non-TEE reports showing that the alterations in Doppler flow velocity waveforms in the fetal descending aorta can identify adverse blood indicating fetal descending aortic flow. The fetal descending aortic flow velocity was converted to fetal heart rate by dividing $60 \mathrm{sec}$ by the peak-to-peak time signal $(0.428$ sec $)$; i.e., $60 / 0.468=128$ beats. $\min ^{-1}$

flow redistribution and consequent fetal compromise. ${ }^{3-5}$ It is possible that maternal TEE assessment of fetal descending aortic blood flow could also help prevent intraoperative hypoxia and subsequent intrauterine growth retardation. In the present case, we used TEE in real time to detect any significant decrease in fetal descending aortic flow velocity. This was an indication of low maternal blood perfusion that potentially led to fetal hypoxia as reflected by rapid changes in FHR. Accordingly, the intervention we used was to raise and maintain the temperature and 
maternal perfusion pressure. We found this approach to be both a practical and effective new use of TEE. In our view, this novel use of TEE could potentially be useful in these rare cases of cardiac surgery during pregnancy to recognize and help reduce fetal morbidity and mortality.

Conflicts of interest None declared.

Supporting Fund National Science and Technology Support Program (ID: 2011BAI11B22).

Open Access This article is distributed under the terms of the Creative Commons Attribution-NonCommercial 4.0 International License (http://creativecommons.org/licenses/by-nc/4.0/), which permits any noncommercial use, distribution, and reproduction in any medium, provided you give appropriate credit to the original author(s) and the source, provide a link to the Creative Commons license, and indicate if changes were made.

\section{References}

1. Arnoni RT, Arnoni AS, Bonini RC, et al. Risk factors associated with cardiac surgery during pregnancy. Ann Thorac Surg 2003; 76: 1605-8.

2. Patel A, Asopa S, Tang AT, Ohri SK. Cardiac surgery during pregnancy. Tex Heart Inst J 2008; 35: 307-12.

3. Hecher $K$, Campbell $S$, Doyle P, Harrington $K$, Nicolaides $K$. Assessment of fetal compromise by Doppler ultrasound investigation of the fetal circulation: Arterial, intracardiac, and venous blood flow velocity studies. ACC Current Journal Review 1996; 5: 40-1.

4. Griffin D, Bilardo $K$, Masini $L$, et al. Doppler blood flow waveforms in the descending thoracic aorta of the human fetus. $\mathrm{Br}$ J Obstet Gynaecol 1984; 91: 997-1006.

5. Jouppila $P$, Kirkinen $P$. Increased vascular resistance in the descending aorta of the human fetus in hypoxia. $\mathrm{Br} \mathrm{J}$ Obstet Gynaecol 1984; 91: 853-6. 